\title{
Detecting “different”: Pyrin senses modified GTPases
}

\author{
Cell Research (2014) 24:1286-1287. doi:10.1038/cr.2014.101; published online 5 August 2014
}

\section{Pathogenic bacteria secrete effec- tor proteins that target host cell Rho GTPases to manipulate the actin cy- toskeleton. A recent study in Nature identifies the Pyrin inflammasome as a sensor of this pathogenic process.}

The ability to detect the presence of bacteria is crucial for life in multicellular organisms. While trillions of bacteria constitutively colonize many locations both on and inside the host, the vast majority of these pose no immediate threat. To avoid unnecessary use of resources and collateral tissue damage, the host has to carefully assess which bacteria defensive responses should be initiated. How the immune system distinguishes between pathogenic and non-pathogenic bacteria has been one of the most intriguing questions in immunology.

Mammalian species use two major classes of receptors to sense microbes. Toll-like Receptors (TLRs) detect highly conserved microbial structures, such as lipopolysaccharide (LPS), lipopeptides and DNA [1]. These microbial patterns are present in virtually all bacteria and, by themselves, are not informative for the immunological decision "pathogen or non-pathogen". However, as TLRs are optimized to sense microbes in the extracellular environment (or through endocytosed "sampling" of the environment), activation of TLRs in locations that are normally sterile can inform the immune system of unwanted bacterial presence. Thus, by incorporating "location" as an additional parameter, TLR activation can be utilized to detect signs of pathogens.

In contrast to TLRs, the second major class of innate sensors, which includes the Nod-like Receptors (NLRs), AIM2like Receptors (ALRs), and Rig-I-like Receptors (RLRs), appear to have evolved to respond more selectively to pathogenic microbes [2]. Localized in the cytosol, these receptors survey the inside of the cell for pathogenassociated molecules or activities. Upon detection of such signals, many of these receptors initiate the formation of a large multiprotein complex referred to as the inflammasome, which enables the activation of Caspase-1, pyroptosis, and the secretion of IL- $1 \beta$ and IL- 18 .

Unlike harmless commensals, pathogenic bacteria employ a wide variety of mechanisms to affect the host, including attachment to or invasion of host cells and secretion of toxins. Inflammasomeinducing receptors evolved two distinct approaches to sense these various bacterial virulence strategies. The first approach involves detections of the presence of intracellular microbial patterns ("pattern-triggered immunity"); while these static signals, like TLR ligands, are often present in both commensals and pathogens, their presence in the cytosol indicates an immediate danger. Examples of "pattern-triggered immunity" in the cytosol include: NOD1- and NOD2-mediated detection of intracellular peptidoglycan; AIM2-mediated detection of DNA from replicating or lysed intracellular bacteria; NLRC4mediated detection of intracellular flagellin and components of Type III Secretion Systems released from invading or invaded bacteria; and NLRP3mediated sensing of cytosolic LPS via Caspase-11 (Figure 1). The second approach revolves around the detection of dynamic signals ("effector-triggered immunity"). Here, instead of sensing the pathogen directly, the immune system senses the effects of common virulence mechanisms employed by pathogens. For instance, NLRP3 detects membrane disruption by pore-forming toxins, such as aerotoxin and nigericin; NLRP1b senses proteolytic activity of the Bacillus anthracis anthrax toxin that inactivates MAP kinases; and NOD1 senses the activation of Rho GTPases by Salmonella Typhimurium [2, 3].

In a recent study published in Nature, $\mathrm{Xu}$ et al. [4] describe the sensing of another pathogen-associated dynamic signal: the inactivation of Rho GTPases, which are molecular switches that regulate actin dynamics, by bacterial toxins. The authors identify Pyrin as the receptor responsible for detecting Rho GTPase inactivation. Mutations in the Pyrin-encoding gene $M E F V$ have long been known to be the cause of the autoinflammatory disease familial Mediterranean Fever; the ligand for MEFV, however, had remain elusive until now. Using an array of Rho GTPase-targeting toxins from multiple bacteria, including the Clostridium difficile TcdB toxin and the C. botulinum $\mathrm{C} 3$ toxin, the authors show that Pyrin senses the inactivation of $\mathrm{RHOA} / \mathrm{B} / \mathrm{C}$, but not other Ras GTPases. Since a variety of toxin-mediated Rho GTPase modifications, including glucosylation, adenylylation, ADP-ribosylation and deamidation, could trigger Pyrin activation, and no direct binding of the modified GTPases to Pyrin was observed, the authors conclude that Pyrin likely senses the effects of GTPase inactivation on the actin cytoskeleton rather than a specific modification itself. 


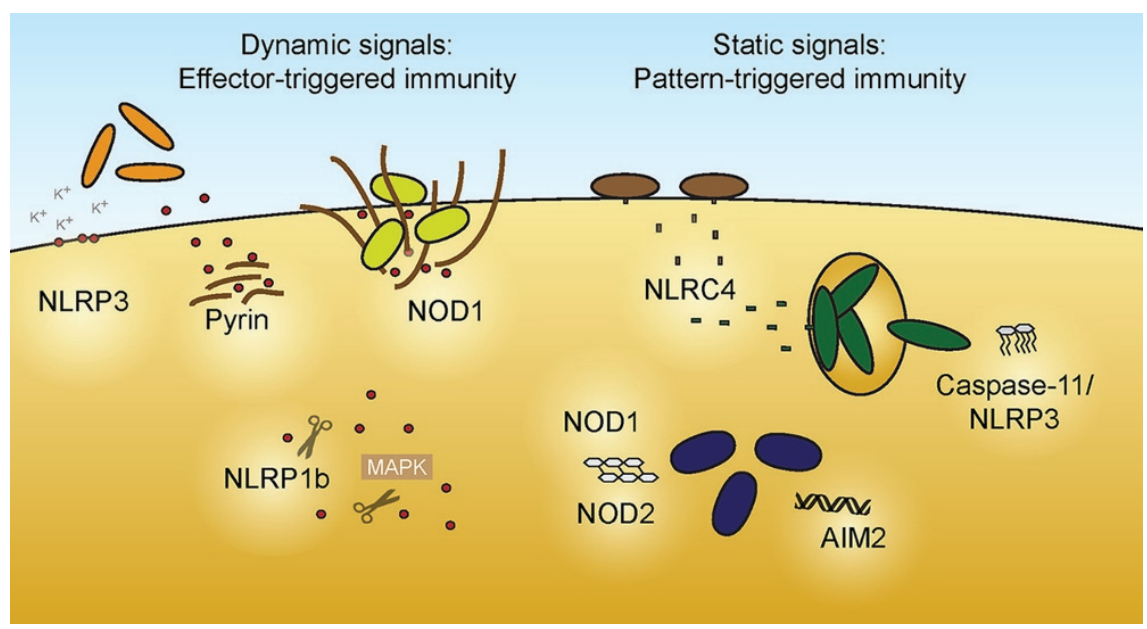

Figure 1 Effector-triggered and pattern-triggered immunity in mammalian cells. Bacterial effectors (shown in red) gain access to the cytosol, where they manipulate cellular processes through pore formation in the cellular membrane (sensed by NLRP3), GTPase inactivation (sensed by Pyrin), GTPase activation (sensed by NOD1), or MAPK inactivation (sensed by NLRP1b). Bacterial patterns are released or secreted from attaching or invading bacteria, where they are detected by NLRC4 (sensing flagellin and components of the Type III Secretion System), NOD1 and NOD2 (sensing peptidoglycan), AIM2 (sensing DNA), and Caspase-11/NLRP3 (sensing LPS).

Because Rho GTPases control multiple fundamental cellular processes including cell motility, cytoskeletal morphology, and cell growth, they are highly attractive targets for manipulation by pathogenic bacteria $[5,6]$. Both inactivation and activation of Rho GTPases by bacterial toxins or effector proteins have been well described; the former often results in inhibition of (immune) cell migration, disruption of the actin cytoskeleton and eventually cell death, while the latter often is utilized by bacteria to gain access into the cytosol. With the discovery of Pyrin-mediated detection of GTPase inactivation, and the recently described NOD1-mediated sensing of GTPase activation [3], both types of "unnatural" actin dynamics are now known to be patrolled by the innate immune system. While Pyrin displays strict specificity for RHOA/B/C, NOD1 was shown to be more promiscuous and could detect activated RHOA, Rac1 and $\mathrm{Cdc} 42$.

The findings by $\mathrm{Xu}$ et al. also raise several interesting questions. For instance, what exactly does Pyrin sense? As the receptor lacks a ligand-binding leucine-rich repeat domain, elucidating the function of the C-terminal B30.2 domain, which has been reported to bind actin and to facilitate co-localization with the cytoskeleton [7], will likely shed light on Pyrin's activation mechanism. Also, it will also be interesting to investigate whether certain pathogenic bacteria intentionally activate Pyrinmediated inflammatory responses to provide a competitive advantage over resident commensals, as has been described for the inflammation induced during S. Typhimurium infection [8]. Finally, while a previous report has de- scribed that TcdB toxin from $C$. difficile activated an unknown inflammasome [9], this study showed that the enzymatic activity of TcdB was dispensable. Further investigation will be required to clarify this discrepancy.

"Effector-triggered immunity" has long been recognized as a central mechanism of immune-mediated detection of pathogens in plants [10]. The discovery that Pyrin can mediate a version of "effector-triggered immunity" in mice highlights the emerging similarities between pathogen detection by inflammasomes in mammals and "effectortriggered immunity" in plants. Future studies may reveal novel "effectortriggered immunity"-related functions for the remaining orphan NLRs.

\section{Marcel R de Zoete ${ }^{1,2}$,} Richard A Flavell ${ }^{1,2}$

${ }^{1}$ Department of Immunobiology, Yale University School of Medicine, New Haven, CT 06520, USA; ${ }^{2}$ Howard Hughes Medical Institute, Yale University, New Haven, CT 06520, USA

Correspondence: Richard A Flavell

E-mail: richard.flavell@yale.edu

\section{References}

1 Palm NW, Medzhitov R. Immunol Rev 2009; 227:221-233

2 Latz E, Xiao TS, Stutz A. Nat Rev Immunol 2013; 13:397-411.

3 Keestra AM, Winter MG, Auburger JJ, et al. Nature 2013; 496:233-237.

4 Xu H, Yang J, Gao W, et al. Nature 2014; 513:237-241.

5 Aktories K. Nat Rev Microbiol 2011; 9:487498.

6 Lemichez E, Aktories K. Exp Cell Res 2013; 319:2329-2336.

7 Mansfield E, Chae JJ, Komarow HD, et al. Blood 2001; 98:851-859.

8 Winter SE, Keestra AM, Tsolis RM, et al. Cell Host Microbe 2010; 8:36-43.

$9 \mathrm{Ng} \mathrm{J}$, Hirota SA, Gross O, et al. Gastroenterology 2010; 139:542-552, 552.e541-e543.

10 Jones JD, Dang1 JL. Nature 2006; 444:323329. 